\title{
Influence of resistance breeding in common bean on rhizosphere microbiome composition and function
}

\author{
Lucas William Mendes ${ }^{1,2}$, Jos M Raaijmakers ${ }^{2,3}$, Mattias de Hollander², Rodrigo Mendes ${ }^{4}$ \\ and Siu Mui Tsai ${ }^{1}$ \\ ${ }^{1}$ Cell and Molecular Biology Laboratory, Center for Nuclear Energy in Agriculture CENA, University of Sao \\ Paulo USP, Piracicaba, Brazil; ${ }^{2}$ Department of Microbial Ecology, Netherlands Institute of Ecology NIOO- \\ KNAW, Wageningen, The Netherlands; ${ }^{3}$ Institute of Biology, Leiden University, Leiden, the Netherlands and \\ ${ }^{4}$ Embrapa Meio Ambiente, Jaguariuna, Brazil
}

\begin{abstract}
The rhizosphere microbiome has a key role in plant growth and health, providing a first line of defense against root infections by soil-borne pathogens. Here, we investigated the composition and metabolic potential of the rhizobacterial community of different common bean (Phaseolus vulgaris) cultivars with variable levels of resistance to the fungal root pathogen Fusarium oxysporum (Fox). For the different bean cultivars grown in two soils with contrasting physicochemical properties and microbial diversity, rhizobacterial abundance was positively correlated with Fox resistance. Pseudomonadaceae, bacillaceae, solibacteraceae and cytophagaceae were more abundant in the rhizosphere of the Fox-resistant cultivar. Network analyses showed a modular topology of the rhizosphere microbiome of the Fox-resistant cultivar, suggesting a more complex and highly connected bacterial community than in the rhizosphere of the Fox-susceptible cultivar. Metagenome analyses further revealed that specific functional traits such as protein secretion systems and biosynthesis genes of antifungal phenazines and rhamnolipids were more abundant in the rhizobacterial community of the Fox-resistant cultivar. Our findings suggest that breeding for Fox resistance in common bean may have co-selected for other unknown plant traits that support a higher abundance of specific beneficial bacterial families in the rhizosphere with functional traits that reinforce the first line of defense.
\end{abstract} The ISME Journal (2018) 12, 212-224; doi:10.1038/ismej.2017.158; published online 13 October 2017

\section{Introduction}

The rhizosphere microbiome, that is, the totality of microorganisms, their genomes and interactions in the rhizosphere, has been the focus of numerous studies to resolve its role in plant growth and health (Mendes et al., 2013; Philippot et al., 2013; Berg et al., 2014). Microorganisms inhabiting the rhizosphere may provide a range of beneficial functions for the host plant, in particular nutrient acquisition, stress tolerance and protection against soil-borne pathogens (Mendes et al., 2011, 2014; PérezJaramillo et al., 2015). The bulk soil is the main source of microbial species colonizing the rhizosphere, and the plant genotype drives, in part, the selection of the microorganisms by depositing specific exudates in the soil-root interface (Jones et al., 2009; Mendes et al., 2013). Hence, plant species, cultivars and soil type are key drivers of

Correspondence: LW Mendes, Cell and Molecular Biology Laboratory, Center for Nuclear Energy in Agriculture CENA, University of Sao Paulo USP, Avenida Centenario 303, Piracicaba, Sao Paulo 13400-970, Brazil.

E-mail: lucaswmendes@gmail.com

Received 16 September 2016; revised 21 June 2017; accepted 24 June 2017; published online 13 October 2017 rhizosphere microbiome composition and functioning (Marschner et al., 2001; Berg and Smalla, 2009; Bulgarelli et al., 2012, 2015; Inceoglu et al., 2012).

Over the past decades, plant breeders have exploited plant genetic traits to improve plant growth and tolerance to biotic and abiotic stresses (Pérez-Jaramillo et al., 2015). Despite the increasing knowledge about the beneficial role of the rhizosphere microbiome for plants, there are only few examples of breeding programs that have considered rhizosphere-related traits (Smith and Goodman, 1999; Smith et al., 1999; Wissuwa et al., 2009). In this context, Bakker et al. (2012) stated that future breeding programs should evaluate plant lines for their broad interaction with soil microorganisms. Previous studies on the microbial basis of disease suppressive soils already showed that plants rely, at least in part, on the rhizosphere microbiome as a first line of defense against pathogen invasion (Mendes et al., 2011; Chapelle et al., 2015; Raaijmakers and Mazzola, 2016). A recent study has shown that the phytohormone salicylic acid modulates colonization of Arabidopsis roots by specific bacterial taxa, illustrating how plant defense pathways impact on the rhizosphere microbiome composition (Lebeis et al., 2015). This study provided, for the first time, 
evidence of a connection between the plant immune system and the rhizosphere microbiome composition. Yao and Wu (2010) previously showed that the rhizobacterial community composition of a cucumber cultivar resistant to Fusarium oxysporum (Fox) differed from that of susceptible cultivars. Whether disease resistance also affects rhizosphere microbiome assembly of other crop species and, more importantly, the functional potential of the rhizosphere microbiome is yet unknown.

Here, we investigated how breeding for resistance of common bean (Phaseolus vulgaris) to the root pathogen FOX influences rhizosphere microbiome assembly and the abundance of specific functional traits associated with the first line of defense provided by the rhizosphere microbiome. Genetic resistance in common bean to Fox is based on a pool of genes with epistasis (Mukankusi et al., 2011) and nine quantitative trait loci were found significantly associated with Fox resistance (Román-Avilés and Kelly, 2005). The resistance is quantitatively inherited and influenced by environmental conditions (Baggett et al., 1965; Schneider and Kelly, 2000). Combining 16S ribosomal RNA (rRNA) amplicon sequencing and shotgun metagenomics, we assessed the taxonomical and functional profile of the rhizosphere microbiome associated with four common bean cultivars, with different levels of Fox resistance, growing in two soils with contrasting physicochemical properties and microbial diversity. This approach allowed us to investigate the effects of soil type and host genotype on rhizosphere microbiome composition, and to identify potential beneficial microbial groups and functional traits affected by resistance breeding.

\section{Materials and methods}

Soil sampling and physicochemical parameters

In order to test the effect of soil properties and diversity on the rhizosphere microbiome we conducted mesocosm experiments with two contrasting soil types specially related to microbial diversity, that is, Amazon Dark Earth (ADE) and an agricultural soil (AGR). The ADE soil was collected at the Hatahara site, located within the Amazon basin near Iranduba-Manaus, Brazil ( $03^{\circ} 16^{\prime} \mathrm{S}$ and $\left.60^{\circ} 12^{\prime} \mathrm{W}\right)$. The ADE soils are anthropogenic horizons built-up by the Pre-Colombian Indians between 500 and 8700 years ago, and they are characterized by their high fertility and high microbial diversity (Brossi et al., 2014). The AGR was collected in an experimental area of the 'Luiz de Queiroz' College of Agriculture (ESALQ/ USP, Piracicaba, Brazil), located at Anhembi muni-

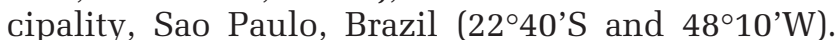
The AGR soil is characterized as oxisol and this area has been used for agricultural experiments with common bean and corn. Soil physical and chemical properties were determined in triplicate for $\mathrm{ADE}$ and AGR based on $400 \mathrm{~g}$ of soil, performed at the
Laboratory of Soil Analysis at ESALQ/USP according to the methodology described by Camargo et al. (2009). In brief, soil pH was measured in a 1:2.5 soil/ water suspension. Exchangeable $\mathrm{Al}, \mathrm{Ca}$ and $\mathrm{Mg}$ were extracted with $\mathrm{KCl} 1 \mathrm{M}$. Calcium and Magnesium were determined by atomic absorption spectrometry and Al by acid-base titration. Phosphorus and $\mathrm{K}$ were extracted by ion-exchange resin. Potential acidity $(\mathrm{H}+\mathrm{Al})$ was calculated based on the $\mathrm{pH}$ determined in SMP buffer solution ( $\mathrm{pH}$ SMP). Mehlic 1 was used to extract the available micronutrients (Fe, $\mathrm{Cu}, \mathrm{Mn}$ and $\mathrm{Zn}$ ) being determined by atomic absorption spectrometry. Hot water was used to extract boron, being further determined by spectrophotometry with azomethine-H at $420 \mathrm{~nm}$. The results of macro and micronutrients allowed the calculation of exchangeable bases (SB), the sum of $\mathrm{Ca}, \mathrm{Mg}$, and $\mathrm{K}$; cation exchange capacity, the sum of $\mathrm{Ca}, \mathrm{Mg}, \mathrm{K}, \mathrm{Al}$ and $\mathrm{H}$; base saturation (V), the percentage relation between SB and cation exchange capacity; and $\mathrm{Al}$ saturation $(\mathrm{m} \%)$, the percentage relation between exchangeable $\mathrm{Al}$ and cation exchange capacity. The texture of the soil samples was determined using Bouyoucos densimeter after shaking the soil vigorously with $\mathrm{NaOH} 1 \mathrm{M}$ as dispersant. Total nitrogen was determined by Kieldahl method and $\mathrm{NH}_{4}+$ and $\mathrm{NO}_{3}-$ by Raney/ Kieldahl.

Plant material, experimental design and sampling Soil samples previously collected at the field were used to grow common bean plants in a mesocosm experiment conducted in greenhouse at CENAUniversity of Sao Paulo (USP), Piracicaba, Brazil. Four common bean cultivars were used in the mesocosms. The choice of the common bean cultivars was based on levels of genetic resistance to Fox (Supplementary Table 1), and the seeds were provided by the Agronomic Institute of Campinas (IAC, Campinas, Sao Paulo). The mesocosms were assembled in ceramic pots $(30 \mathrm{~cm}$ high $\times 20 \mathrm{~cm}$ diameter) with a stone layer of $5 \mathrm{~cm}$ on the bottom. Approximately $8 \mathrm{~kg}$ of soil were used to fill the pots, and four seeds were sowed in each pot. Each cultivar was grown in three independent pots per soil type, that is, four common bean cultivars $\times 2$ soil types $\times 3$ pots (replicates), resulting in 24 samples (independent pots). The plants germinated at $28 / 19^{\circ} \mathrm{C}$ (day/ night) with $12 \mathrm{~h}$ photoperiod. The moisture and temperature were regularly adjusted for optimal growth conditions for the plants. Plants were collected at R1 stage (early flower) and the roots with attached soil were removed from the pots and transported on ice to the laboratory. The roots were shaken to remove the loosely adhering soil. The soil attached firmly to the roots was collected with sterile brushes and considered to be the rhizosphere soil. Soil samples collected prior to the bean cultivation, without the effect of common bean roots, were considered as initial soil community and called here 
as 'soil' treatment. For each pot we obtained a single DNA sample, which was used for both shotgun metagenome and $16 \mathrm{~S}$ rRNA sequencing.

\section{DNA extraction}

Total DNA was extracted from $250 \mathrm{mg}$ of soil (soil and rhizosphere) using the PowerLyzer PowerSoil DNA Isolation Kit (MoBio Laboratories, Carlsbad, CA, USA), according to the manufacturer's protocol. Measurements of DNA quality and quantity were performed by $1 \%$ sodium boric acid (Brody and Kern, 2004) agarose gel electrophoresis and NanoDrop 1000 spectrophotometry (Thermo Scientific, Waltham, MA, EUA). The same DNA sample was used for DNA shotgun sequencing and $16 \mathrm{~S}$ rRNA sequencing.

\section{Quantitative PCR analysis}

Quantitative PCR analysis (qPCR) was performed to quantify the number of bacterial gene copies in soil and rhizosphere samples of the four bean cultivars. The amplification reactions were run with universal primers for Bacteria U968F (5'-AACGCGAAGAACC TTAC-3') and R1387 (5'-CGGTGTGTACAAGGCCC GGGAACG-3') (Heuer et al., 1997), which generated amplicons size of $419 \mathrm{bp}$. The reactions were performed in a final volume of $10 \mu$ l containing $5 \mu \mathrm{l}$ of SYBR Green Rox qPCR Kit (Fermentas, Brazil), $2.5 \mu \mathrm{M}$ of each primer and $10 \mathrm{ng}$ of DNA template. DNA amplification was performed with an initial denaturation temperature at $94^{\circ} \mathrm{C}$ for $10 \mathrm{~min}$, followed by 40 cycles of $94^{\circ} \mathrm{C}$ for $30 \mathrm{~s}, 56^{\circ} \mathrm{C}$ for $30 \mathrm{~s}$ and $72^{\circ} \mathrm{C}$ for $40 \mathrm{~s}$ with a final melting curve of $95^{\circ} \mathrm{C}$ for $15 \mathrm{~s}, 56^{\circ} \mathrm{C}$ for $1 \mathrm{~min}$ and temperature increasing to $95^{\circ} \mathrm{C}$ for $15 \mathrm{~s}$, with data reading at each $0.7^{\circ} \mathrm{C}$. Reactions were carried out on the equipment StepOnePlus (Applied Biosystems, Foster City, CA, USA). The Cts values (cycle threshold) were used as standards for determining the amount of DNA template in each sample. Standard curves were produced for the 16S rRNA using cloned fragments of Pseudomonas fluorescens DSM 8369. Gene fragments were quantified in a spectrophotometer (190 and $840 \mathrm{~nm}-$ NanoDrop ND-1000) and diluted (10 $10^{3}$ genes $\left.\mu l\right)$ to generate the standard curve. The gene copy number in different samples was expressed as log copy numbers of gene per gram of soil. Statistical data analyses were performed using One-way analysis of variance and Tukey's test to determine the significance of differences between all soil samples, using the PAST 3 software (Hammer et al., 2001).

$16 S$ rRNA gene sequencing, data processing and taxonomical affiliation

For taxonomical profiling of the bacterial communities, triplicate PCR reactions of a total of 30 samples $((4$ bean cultivars + soil $) \times 3$ replicates $\times 2$ soils) targeting the V3-V4 region of the 16S rRNA gene were performed. The amplification was conducted with the primers S-D-Bact-0341-b-17 (5'-CCTACGGGNGGCWGCAG-3') and S-D-Bact-0785a-A-21 (5'-GACTACHVGGGTATCTAATCC-3'), with an amplicon size of $464 \mathrm{bp}$ (Klindworth et al., 2013). An overhang adapter sequence was added to the locus-specific primers as recommended by Illumina, San Diego, CA, USA. For amplification, the $20-\mu l$ reaction mixtures contained $2 \mu l$ of reaction buffer $\times 10$ (Invitrogen, Carlsbad, CA, USA), $1 \mu \mathrm{l}$ of $\mathrm{MgCl}_{2}$ (50 mM), $1 \mu \mathrm{l}$ of the primer set ( $5 \mathrm{pmol}$ each), $1 \mu \mathrm{l}$ of deoxyribonucleotide triphosphate mixture (2.5 mM each), $0.25 \mu \mathrm{l}$ of Bovine Serum Albumin $\left(1 \mathrm{ng} \mathrm{ml}^{-1}\right), \quad 0.2 \mu \mathrm{l}$ (5 U) of Platinum Taq DNA Polymerase (Invitrogen), $1 \mu \mathrm{l}$ of DNA template (20 ng) and $12.55 \mu \mathrm{l}$ of sterilized ultrapure water. PCR amplification was performed using a GeneAmp PCR System 9700 Thermal Cycler (Applied Biosystems) with the following amplification cycles: $95^{\circ} \mathrm{C}$ for $5 \mathrm{~min}$, followed by 25 cycles of $95^{\circ} \mathrm{C}$ for $40 \mathrm{~s}, 56^{\circ} \mathrm{C}$ for $1.5 \mathrm{~min}$, and $72^{\circ} \mathrm{C}$ for $1 \mathrm{~min}$ with a final extension step at $72^{\circ} \mathrm{C}$ for $7 \mathrm{~min}$. Negative and positive controls were run in all amplifications. Products were purified with Agencourt AMPure XP reagents (Beckman Coulter, Brea, CA, USA), on a magnetic rack, as manufacturer's instructions. The purified amplicons were used for the insertion of barcoding indexes with the Nextera XT Index Kit (Illumina). A combination of $5 \times 6$ indexes was used totaling 30 samples per sequencing run. For each PCR, the $50-\mu l$ reaction mixtures contained $5 \mu \mathrm{l}$ of reaction buffer $\times 10$ (Invitrogen), $1.5 \mu \mathrm{l}$ of $\mathrm{MgCl}_{2}$ $(50 \mathrm{~mm}), 5 \mu \mathrm{l}$ of both forward and reverse indexes, $2 \mu \mathrm{l}$ of deoxyribonucleotide triphosphate mixture (2.5 mM each), $0.3 \mu \mathrm{l}$ (5 U) of Platinum Taq DNA Polymerase (Invitrogen), $20 \mu \mathrm{l}$ of purified PCR products and $11.2 \mu \mathrm{l}$ of sterilized ultrapure water. PCR amplification was performed using a GeneAmp PCR System 9700 Thermal Cycler (Applied Biosystems) with the following amplification cycles: $72^{\circ} \mathrm{C}$ for $3 \mathrm{~min}, 95^{\circ} \mathrm{C}$ for $30 \mathrm{~s}$, followed by 12 cycles of $95^{\circ}$ $\mathrm{C}$ for $10 \mathrm{~s}, 55^{\circ} \mathrm{C}$ for $30 \mathrm{~s}$, and $72^{\circ} \mathrm{C}$ for $30 \mathrm{~s}$ with a final extension step at $72^{\circ} \mathrm{C}$ for $5 \mathrm{~min}$. The PCR products were pooled in one single sample and gel purified with GFX PCR DNA and Gel Band Purification Kit (GE Healthcare, Little Chalfont, Buckinghamshire, UK). Prior to sequencing, the pooled purified sample was quantified with the KAPA SYBR FAST Universal qPCR Kit (Kapa Biosystems, Woburn, MA, USA) in a StepOnePlus thermocycler (Thermo Fisher Scientific, Waltham, MA, USA) according to manufacturer's protocol. The pairedend sequencing was performed with Miseq Reagent Kit v3 (600 cycles; Illumina) in a Miseq Personal Sequencing System (Illumina).

The data obtained by the sequencing was analyzed with bioinformatics tools as follow. Initially, primer sequences were removed from the per sample FASTQ files using Flexbar version 2.5 (Dodt et al., 2012). All reads were trimmed to a minimum length 
of $150 \mathrm{bp}$ and at least a Phred score of 25 by using fastq-mcf (https://expressionanalysis.github.io/eautils/). The remaining sequences were converted to FASTA format and concatenated into a single file. All reads were clustered into OTUs using the UPARSE strategy by dereplication, sorting by abundance with at least two sequences and clustering using the UCLUST smallmem algorithm (Edgar, 2010). These steps were performed with VSEARCH version 1.0.10 (Rognes et al., 2015), which is an open-source and 64-bit multithreaded compatible alternative to USEARCH. Next, chimeric sequences were detected using the UCHIME algorithm (Edgar et al., 2011) implemented in VSEARCH. All reads were mapped before the dereplication to OTUs using the usearch_global method implemented in VSEARCH to create an OTU table and converted to BIOM-Format 1.3.1 (McDonald et al., 2012). Finally, taxonomic information for each OTU was added to the BIOM file by using the RDP Classifier version 2.10 (Cole et al., 2014). All steps were implemented in a Snakemake workflow (Köster and Rahmann, 2012) as shown in Supplementary Figure 1. Singletons and doubletons, mitochondrion, chloroplast and eukaryotic sequences were removed and the BIOM file generated was used for statistical analyses. The 16S rRNA data are available at EBI (PRJEB14409).

Shotgun metagenomics, data processing and functional annotation

In total, 30 DNA sample libraries ( 4 bean cultivars + soil) $\times 3$ replicates $\times 2$ soils) were prepared using the Miseq Reagent Kit v2 (500 cycles; Illumina), according to the manufacturer's protocol for shotgun metagenomic sequencing in a Miseq Personal Sequencing System (Illumina). The paired-end sequences obtained were merged using FLASH version 1.2.5 (Magoč and Salzberg, 2011) and lowquality bases (quality score lower than 20) from merged and unmerged sequences were trimmed from both ends using the Phred algorithm with SeqyClean script (https://github.com/ibest/seqy clean). Unassembled DNA sequences were annotated with Metagenomics Rapid Annotation (MG-RAST) pipeline version 3.5 (Meyer et al., 2008). The functional profile was generated using the normalized abundance of sequence matches to the SEED database (Aziz et al., 2008). For each metagenome, a table of the frequency of subsystems hits was generated and normalized by dividing by the total number of hits to remove bias indifference in sequencing efforts and read length. To identify hits, BlastX was used with minimum alignment length of $15 \mathrm{bp}$, minimal identity of $60 \%$, and an $E$-value cutoff of $\mathrm{E}<1 \times 10^{-5}$. The data matrices generated were used for statistical analyses. The shotgun metagenome data are available at MG-RAST under the project 'Common Bean Rhizosphere Microbiome'
(ID 12156) (more information on Supplementary

Table 2).

\section{Data analysis}

Redundancy analysis (RDA) was used to visualize the community structure and determine its correlation with environmental parameters. First, the matrices were analyzed using Detrended Correspondence analysis to evaluate the gradient size of the species distribution, which indicated linearly distributed data (length of gradient $<3$ ), suggesting the RDA as the best-fit mathematical model for the data. Forward selection and the Monte Carlo permutation test were applied with 1000 random permutations to verify the significance of environmental parameters upon the microbial community structure. RDA plots were generated using Canoco 4.5 software (Biometrics, Wageningen, The Netherlands). We used permutational multivariate analysis of variance (PERMANOVA) (Anderson, 2001) to test whether sample categories harbored significantly different metagenome or microbial community structures. Alpha diversity was calculated from a matrix of richness using Shannon's index. PERMANOVA and alpha diversity indexes were calculated with the software PAST 3 (Hammer et al., 2001). Phylogenetic diversity was calculated as Faith's PD (Faith, 1992) by using Qiime (Caporaso et al., 2010). Venn diagrams were also constructed to verify the proportion of groups exclusive and shared between samples using the webtool Venny 2.0.2 (Oliveiros, 2007). To determine the statistical differences between the treatments, the Statistical Analysis of Metagenomics Profile v2.1.3 (STAMP) software was used (Parks et al., 2014). For $16 \mathrm{~S}$ profiling, the BIOM file was used as input. For functional profiling a table with hits frequency of the functional subsystems (SEED database) for each metagenome was generated from MG-RAST and used as input. $P$-values were calculated using the two-sided Fisher's exact test (Fisher, 1958), confidence intervals were calculated using the Newcombe-Wilson method (Newcombe, 1998) and correction was made using Benjamini-Hochberg false discovery rate (Benjamini and Hochberg, 1995).

In addition, network analyses were performed to assess the complexity of the interactions among microbial taxa. Non-random co-occurrence analyses were performed using SparCC, a tool capable of estimating correlation values from compositional data (Friedman and Alm, 2012). For this, quality reads were clustered at $97 \%$ identity and an OTU table affiliated at genus level was used for analysis. For each network, $P$-values were obtained by 99 permutations of random selections of the data table, subjected to the same analytical pipeline. Statistically significant $(P<0.01)$ SparCC correlations with a magnitude of $>0.7$ or $<-0.7$ were included into the network analyses. The nodes in the reconstructed network represent taxa at genus level, whereas the edges represent significantly positive or negative 

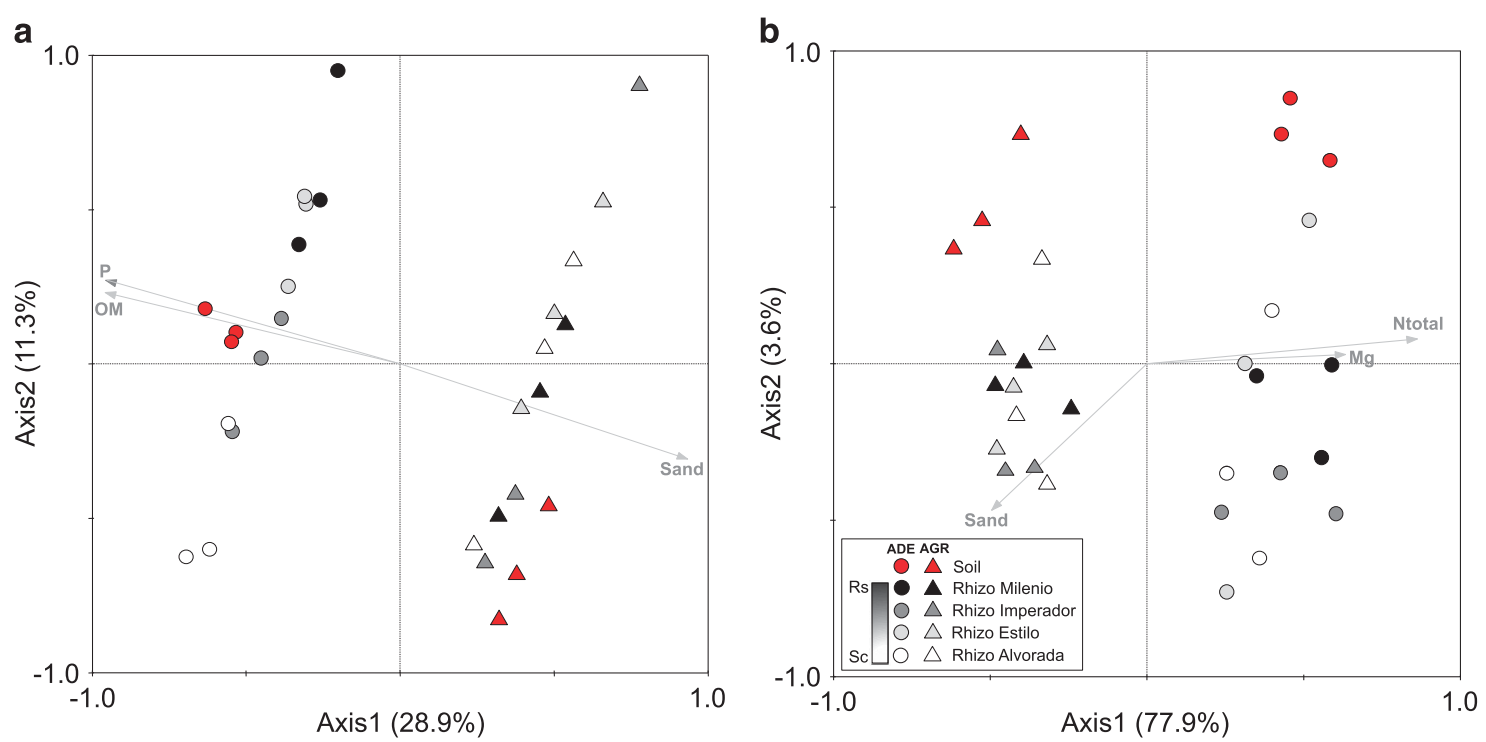

Figure 1 Redundancy analysis (RDA) performed on (a) taxonomic (16S rRNA) and (b) functional (metagenome) profiles and environmental characteristics for two types of soils and rhizosphere of four common bean cultivars. Arrows indicate correlation between environmental parameters and microbial structure. The shown environmental parameters were chosen based on significant correlation evaluated via the Monte Carlo permutation test $(P<0.05)$; Rhizo=rhizosphere soil; Rs = resistant; $\mathrm{Sc}=$ susceptible; $\mathrm{Mg}=\mathrm{Magnesium}$; Ntotal = Total Nitrogen; OM = Organic matter; $\mathrm{P}=$ Phosphorus .

correlations between nodes. The network graphs were made based on a set of measures, as number of nodes, number of edges, modularity, number of communities, average node connectivity, average path length, diameter and cumulative degree distribution. Co-occurrence analyses were carried out using the Python module 'SparCC' and networks visualization and properties measurements were calculated with the interactive platform Gephi (Bastian and Jacomy, 2009).

\section{Results and discussion}

Rhizosphere effect on soil properties and microbial recruitment

The soil is considered as the prime source of microbial species richness and diversity in the plant rhizosphere. Hence, many studies have demonstrated the influence of soil type on the composition and structure of microbial communities in the rhizosphere (Ridder-Duine et al., 2005; Berg and Smalla, 2009; Bulgarelli et al., 2012; Inceoglu et al., 2012; Lundberg et al., 2012; Bakker et al., 2015). The two soil types used in our experiment, Amazon Dark Earth (ADE) and AGR, differed significantly in physicochemical properties (Supplementary Table 3). This contrast was confirmed by molecular analyses, which revealed higher amounts of DNA retrieved and higher bacterial abundance in ADE than in AGR soil (Supplementary Figure 2). For both ADE and AGR soils, cultivation of all four bean cultivars had substantial effects on the physical and chemical properties of the rhizosphere soil, including significant declines in $\mathrm{K}$ and $\mathrm{H}+\mathrm{Al}$ as compared with the soil $(P<0.05)$. However, bean cultivation increased base saturation index as well as $\mathrm{Ca}$ and Fe contents $(P<0.05)$. Overall, the rhizosphere effect was stronger in the AGR soil (Supplementary Tables 4 and 5). These changes are most likely due to several processes occurring at the root-soil interface during plant development, including the release of exudates, uptake of water and nutrients, and root respiration (Philippot et al., 2013). In addition, the nutrient availability in rhizosphere is controlled by interactions among soil properties, plant characteristics, and microbial activity. Although a decrease of iron and calcium in the rhizosphere is common, accumulation owing to exudates and microbial solubilization may occur at some plant developmental stage (Moore et al., 1965; Calba et al., 2004; Rengel, 2015).

In addition to these chemical alterations, the taxonomic composition and functional profile of the bacterial community in the rhizosphere of all bean cultivars was different from that of the bulk soil, exemplifying the well-known rhizosphere effect. RDA of the taxonomic composition resulted in samples clustering firstly according to the soil type (Figure 1a) as confirmed by PERMANOVA $(F=10.35, P=0.0001)$, followed by a separation by sample type, that is, soil or rhizosphere (PERMANOVA-ADE $F=4.937, P=0.0038 ;$ AGR $F=3.035$, $P=0.0026$ ) (Supplementary Table 6; for taxonomic affiliation see Supplementary Figure 3). The taxonomic differences between the common bean cultivars were more evident in ADE soil, where the structure of the rhizosphere communities was significantly different among the cultivars (PERMANOVA $F=2.469, P=0.0007$ ).

For the functional annotation of genes, the samples were first clustered according to the soil type, which 
was explained by the variation of the first axis of the plot $(77.9 \%)$ and confirmed by one-way PERMANOVA ( $F=87.81, P=0.0001$ ) (Figure $1 b)$. Second, the samples were clustered in the second axis of the RDA based on sample type (PERMANOVA $F=1.903$, $P=0.0365$ ). However, it was not possible to discriminate the functional profiles based on the four different cultivars. The forward selection, followed by Monte Carlo permutation test, showed that the structure of the rhizobacterial communities correlates with physicochemical properties such as phosphorus content, organic matter, $\mathrm{Cu}$ and total nitrogen (Figure 1). These parameters were found to be distinctly different between ADE and AGR $(P<0.0005)$.

When the OTUs of each cultivar were compared with those of the bulk soils, we found on average 8.6 and $9.4 \%$ of the OTUs exclusively present in the rhizosphere of beans grown in ADE or AGR, respectively. The core rhizosphere microbiome, that is, the bacterial OTUs shared between all four cultivars, accounted for $\sim 73 \%$ of all OTUs (Supplementary Figure 4A). When the OTUs of the core microbiome were compared with the bulk soil, we found an average of $8.6 \%$ of OTUs present in the rhizosphere of all cultivars that were not found at detectable numbers in the soil (Supplementary Figure 4B). When rhizosphere OTUs were compared among the cultivars, an average of $2.5 \%$ were cultivar-specific. This cultivar specificity was more prominent in the AGR soil, where the Fox-resistant cultivar harbored $4.5 \%$ unique OTUs. The OTUs exclusively present in the rhizosphere of the Foxresistant cultivar were affiliated to 13 phyla, dominated by Actinobacteria $(21.2 \%)$, Bacteroidetes (15.8\%), Verrucomicrobia (15.5\%) and Proteobacteria (11.9), with some variation between ADE and AGR soils (Supplementary Figure 5).

Based on the results of RDA and PERMANOVA, we grouped the samples of rhizosphere and compared the phyla abundance to that in soil. Considering both soil types, five phyla were more abundant in the rhizosphere, namely Armatimonadetes, Chlamydiae, Cyanobacteria, Candidatus Saccharibacteria and Deinococcus-Thermus (Supplementary Figure 6). Some members of these groups have an important role in nutrient cycling. In a previous study, we showed that soybean plants are able to recruit microbial taxa based on the functional cores related to benefits for the plant, such as growth promotion and nutrition (Mendes et al., 2014). Analyzing the functional profile (Supplementary Figure 7A), we detected a high abundance of sequences in the rhizosphere-related to motility and chemotaxis. Flagellar motility, along with quorum sensing, allows the microbial populations to actively access available resources and avoid feeding by predators (Mallon et al., 2015). We also found a high abundance of sequences related to membrane transport. Some membrane transport systems can contribute to the competitiveness of a microbial community, such as Type VI secretion systems (T6SS), whose function is to mediate extracellular export of virulence factors and their translocation into target eukaryotic cells (Leiman et al., 2009). Some of these functions may also hamper pathogen invasion, contributing to the function of the rhizosphere microbiome as a first line of defense. Interestingly, when we analyzed these two functions for each cultivar we found that Fox-resistant cultivars showed a higher abundance of membrane transport genes than Fox-susceptible cultivars in ADE soil and also a higher abundance of motility and chemotaxis genes in AGR soil (Supplementary Figure 7B).

Modulation of the rhizosphere microbiome by the Foxresistant bean cultivar

In order to explore possible correlations between pathogen resistance in plants and the rhizosphere microbiome composition, we selected the most resistant (IAC Milenio) and most susceptible (IAC Alvorada) bean cultivars for detailed analysis. The resistant cultivar IAC Milenio used in our experiments is derived from a cross between two cultivars, one being a sibling line of the susceptible cultivar IAC Alvorada (Carbonell et al., 2014). Although they are genetically related, we hypothesized that even small changes in the genome of the Fox-resistant cultivar may affect rhizosphere community assembly. In the Fox-resistant bean cultivar, the colonization of the fungus between adjacent xylem vessels is restricted by chemical and structural alterations, including vascular occlusion by gel plugs, tyloses, deposition of additional cell wall layers and infusion of these structures with phenols and other metabolites (Mace et al., 1981; Pereira et al., 2013). In a recent study, Beckers et al. (2016) demonstrated that variation in lignin biosynthesis modulates the composition of the plant endosphere microbiome. Considering that plants rely, at least in part, on the rhizosphere microbiome for functions and traits related to growth, development and health (Mendes et al., 2013), we postulated that breeding for Fox resistance in common bean may have unintentionally co-selected for plant traits affecting the recruitment of beneficial cultivar-specific microbiota.

According to richness and both diversity indices used, that is, Shannon and Faith's PD, the Foxresistant cultivar presented higher bacterial community diversity when compared with the bulk soil (Figures 2a-c). Interestingly, the bacterial abundance was higher in the rhizosphere of the Fox-resistant cultivar grown in ADE soil (Figure 2d), and this abundance decreased in the rhizosphere of bean cultivars with increasing susceptibility to the pathogen. For diversity, we could observe a similar trend for both soil types, however, not statistically significant $(P>0.05)$. Higher bacterial abundance found in the rhizosphere of the Fox-resistant cultivar may result in a higher competition for resource 

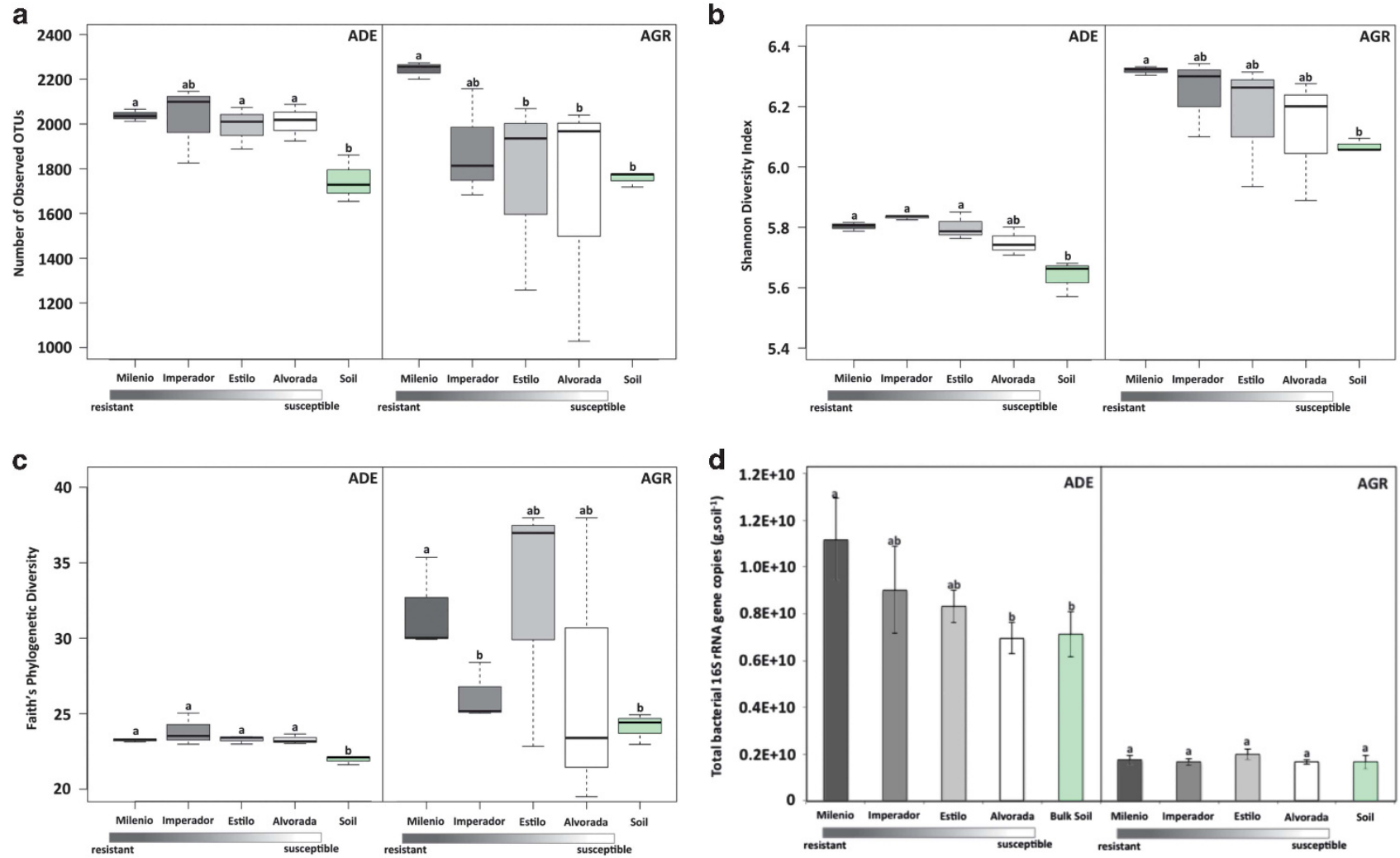

Figure 2 Diversity measurements based of 16S rRNA of soil and rhizosphere microbial communities for Amazon Dark Earth and agricultural soil. (a) Richness based on the number of observed OTUs; (b) phylogenetic diversity calculated as Faith's PD based on 97\% similarity; (c) diversity based on Shannon's index; and (d) abundance of bacterial 16S rRNA gene. Error bars represent the standard deviation of four independent replicates. Different lower case letters refer to significant differences between treatments based on Tukey's test $(P<0.05)$.
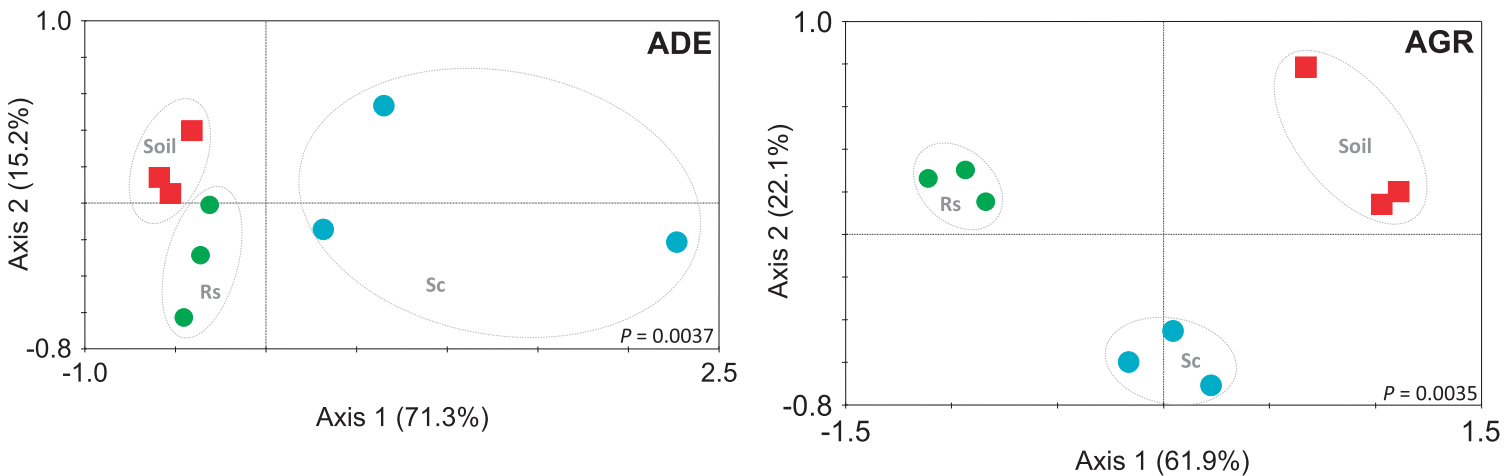

Figure 3 Principal component analysis (PCA) performed on taxonomic profile (OTU level for 16S rRNA data set) for two types of soils and rhizosphere of two common bean cultivars with contrasting levels of resistance to the pathogen Fusarium oxysporum. P-value evaluated via one-way PERMANOVA test. Rs = rhizosphere of the resistant cultivar Milenio; Sc = rhizosphere of the susceptible cultivar Alvorada; Soil = initial soil community; ADE= Amazon Dark Earth; AGR = agricultural soil.

contributing to protection against fungal infection. Resource competition is enhanced in highly abundant and more diverse communities and has been proposed as a key factor for the success or failure of pathogen invasion (Wei et al., 2015).

Comparative analysis of the rhizosphere microbiome between the Fox-resistant and susceptible bean cultivars revealed a distinct community structure and differential abundance of specific family groups (Figures 3 and 4). These results extend the results of Yao and Wu (2010), who demonstrated that the rhizospheric community structure of a cucumber cultivar resistant to $F$. oxysporum differed in comparison to susceptible cultivars. Our 16S rRNA and metagenome sequence data for both soil types further pointed to a higher abundance of the families Pseudomonadaceae, Bacillaceae, Cytophagaceae and Solibacteraceae in the rhizosphere of the Foxresistant cultivar (Figures 4a-d). At a lower taxonomical level, we found higher abundance of the 

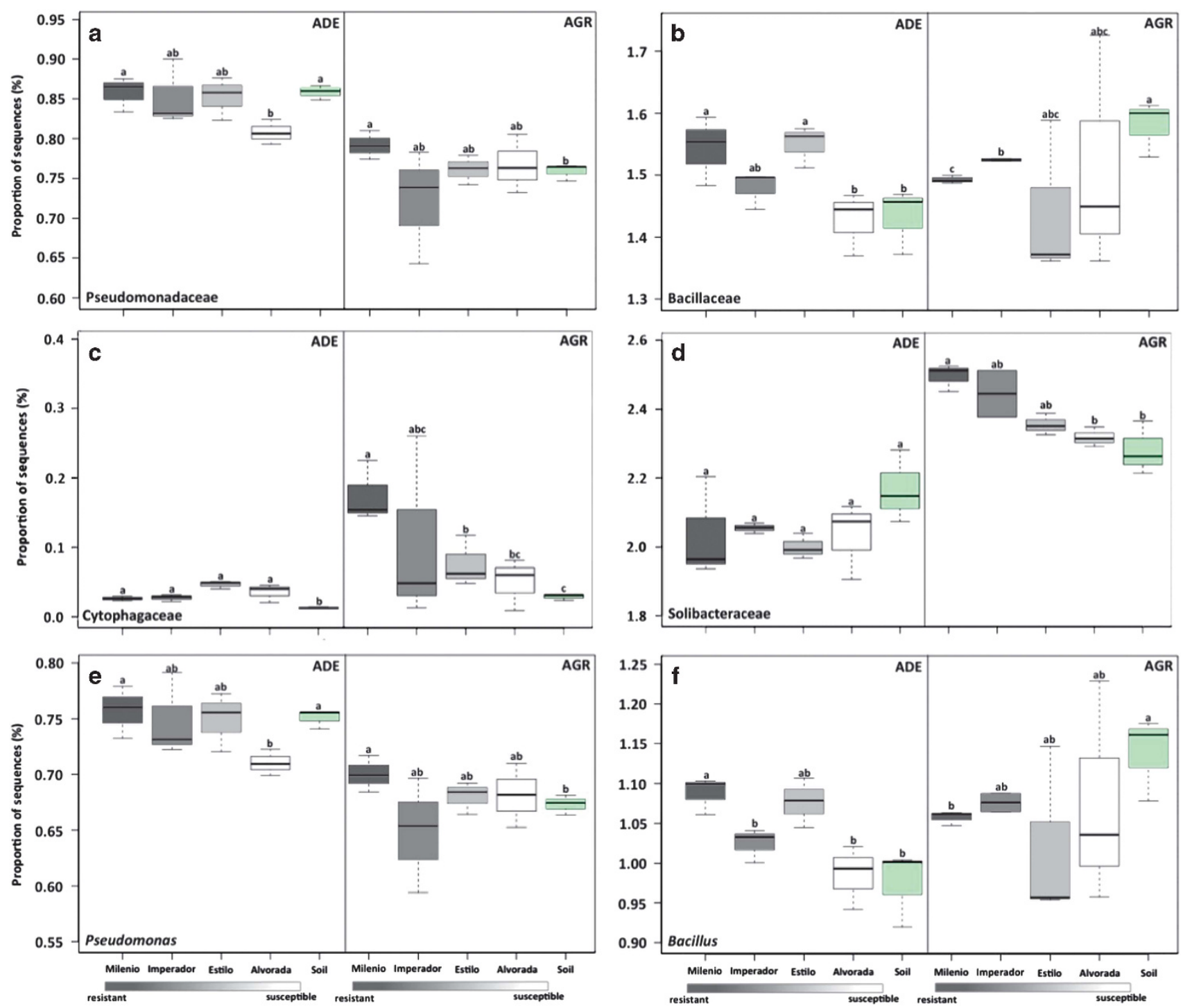

Figure 4 Distribution of the most differential bacterial groups ((a-d) family level and (e-f) genera level, based on 16S rRNA dataset) in the rhizosphere of different common bean cultivars in Amazon Dark Earth (ADE) and agricultural soil (AGR). Boxes indicate IQR (75th to 25th of the data). The median value is shown as a line within the box and outliers are represented by dots. Different lower case letters refer to significant differences between treatments within each soil type based on Tukey's test $(P<0.05)$.

genera Pseudomonas and Bacillus in the rhizosphere of the Fox-resistant cultivar growing in ADE soil (Figures $4 \mathrm{e}$ and f). These two rhizobacterial genera are well-known for biofertilization, stimulation of root growth, rhizo-remediation, control of abiotic stress and plant diseases (Mendes et al., 2013). In a study with common bean, Gilard et al. (2008) showed that Pseudomonas chlororaphis exhibited biological control against fusarium root rot. Also, member of the genus Bacillus are well-known for their ability to protect plants against root pathogens (Cavaglieri et al., 2005). For common bean, Kumar et al. (2012) isolated Bacillus strains from the rhizosphere and showed plant growth promotion and antagonistic activity against several phytopathogens, including Fusarium oxysporum. Based on our metagenome data, sequences assigned to
Pseudomonas and Bacillus were affiliated to nutrient-related metabolism and several antagonistic traits such as phenazine and chitinase biosynthesis, stress response, secondary metabolism, motility, chemotaxis, dormancy and sporulation (Supplementary Table 7). Further exploration of the metagenome data revealed a higher abundance of sequences associated with the biosynthesis of phenazines and rhamnolipids in the rhizosphere of the Fox-resistant cultivar IAC Milenio grown in ADE soil (Figures 5a and b). Phenazine antibiotics have a key role in suppression of $F$. oxysporum on diverse crops (Anjaiah et al., 1998; Chin-a-Woeng et al., 2000). Furthermore, Mazurier et al. (2009) reported that phenazine antibiotics produced by Pseudomonas species contribute to natural soil suppressiveness to fusarium wilt. Rhamnolipids, also produced by Pseudomonas, 

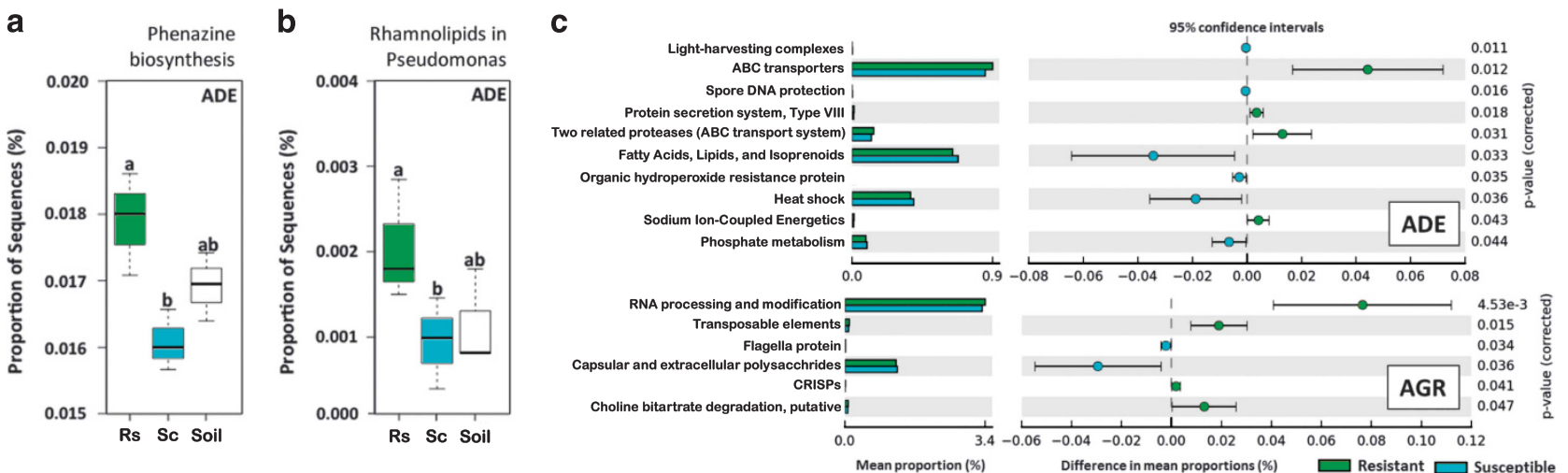

Figure 5 Differential abundance of sequences affiliated to bacterial functions (based on metagenome data set) in the rhizosphere microbiome of resistant and susceptible common bean cultivars grown in two different soils. The sequences were affiliated to functions based on SEED databank (a and b) level 3 and (c) level 2. Resistant=rhizosphere of the resistant cultivar Milenio; Susceptible=rhizosphere of the susceptible cultivar Alvorada; ADE = Amazon Dark Earth; AGR = Agricultural soil.
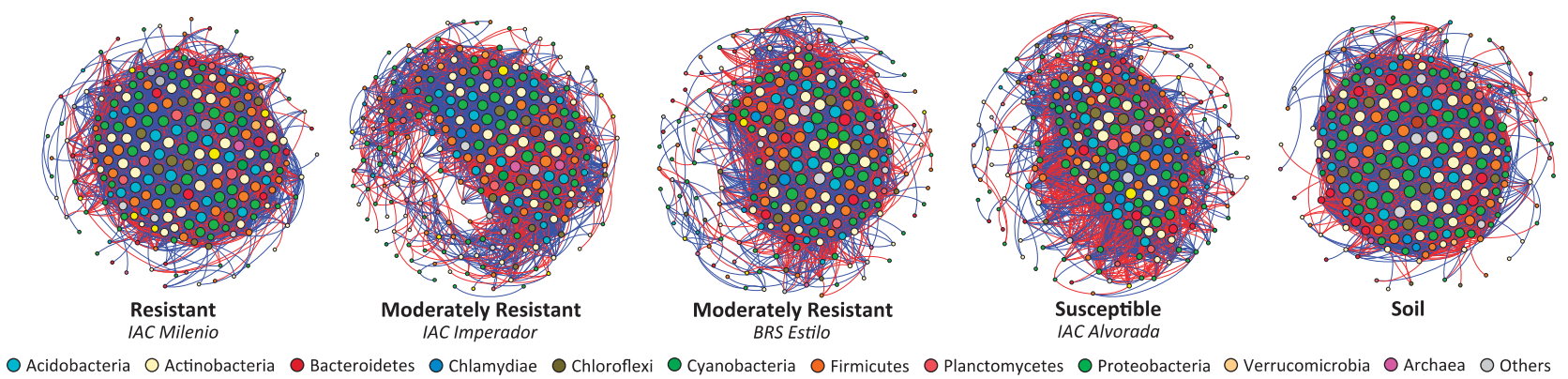

Figure 6 Network co-occurrence analysis of microbial communities of rhizosphere and soil samples. A connection stands for SparCC correlation with magnitude $>0.7$ (positive correlation-blue edges) or $<-0.7$ (negative correlation-red edges) and statistically significant $(P<0.01)$. Each node represents taxa affiliated at genus level (based on $16 \mathrm{~S}$ rRNA), and the size of node is proportional to the number of connections (that is, degree). Each node was labeled at phylum level.

have antimicrobial properties and also act against several fungi including Fusarium (Haba et al., 2003). We also found a higher abundance of sequences affiliated to $\mathrm{ABC}$ transporters and protein secretion system in the rhizobacterial community of the Foxresistant cultivar (Figure 5c). Some of these functions are related to the export of various virulence factors, such as antibiotics, bacteriocins, toxins, that may increase competition within a community hindering pathogen invasion. Therefore, based on our findings we hypothesize that the Fox-resistant cultivar is better able to recruit beneficial microbial groups that, in addition to the plant genetic traits, may complement the defense against pathogen infection.

Network structure of the rhizosphere microbiome We then used co-occurrence network analysis to explore the complexity of connections within the rhizosphere microbiomes of the different common bean cultivars. For this, we calculated SparCC correlations between microbial taxa at genus level based on 16S rRNA data. Following this, we calculated the topological properties of the obtained networks to identify differences between the samples. The Fox-resistant cultivar showed the highest level of complexity and modular structure, whereas the Fox-susceptible cultivar presented a less complex (Figure 6) and less modular network (Table 1). It has been assumed that highly diverse bacterial communities are often more resistant to pathogen invasion (Latz et al., 2012; Mallon et al., 2015). This hypothesis is based on the assumption that diverse communities exhibit a higher number of species interactions and intensified competition for niche space (Kennedy et al., 2002; van Elsas et al., 2012). Although we did not find significant differences in the rhizobacterial diversity between the Fox-resistant and the susceptible bean cultivars, we found a higher complexity in the network of the Fox-resistant cultivar. Wei et al. (2015) suggested that the relationships of diversity-invasion resistance could be mechanistically explained by the underlying interaction network architecture. The network of the Foxresistant cultivar presented the highest number of connections per node (average degree $=84.59$ ), and a lower average path length (1.66) and diameter (6) in comparison with the other cultivars, indicating a highly connected community (Table 1). Average path length is defined as the average number of steps along the shortest paths between each node, being a measure of efficiency on a network (Zhou 
Table 1 Correlations and topological properties of common bean rhizosphere microbiome networks

\begin{tabular}{|c|c|c|c|c|c|}
\hline Network properties & $\begin{array}{l}\text { Resistant IAC } \\
\text { Milenio }\end{array}$ & $\begin{array}{l}\text { Mod. resistant IAC } \\
\text { Imperador }\end{array}$ & $\begin{array}{l}\text { Mod. resistant BRS } \\
\text { Estilo }\end{array}$ & $\begin{array}{c}\text { Susceptible IAC } \\
\text { Alvorada }\end{array}$ & Soil \\
\hline Number of nodes ${ }^{\mathrm{a}}$ & 230 & 253 & 239 & 241 & 222 \\
\hline Number of edges ${ }^{b}$ & 9728 & 5243 & 6474 & 5883 & 9457 \\
\hline Positive edges $^{\mathrm{C}}$ & 5049 & 3124 & 3342 & 3239 & 4831 \\
\hline Negative edges ${ }^{\mathrm{d}}$ & 4679 & 2119 & 3132 & 2644 & 4626 \\
\hline Modularity ${ }^{\mathrm{e}}$ & 12.27 & 2.45 & 14.46 & 4.57 & 18.08 \\
\hline Number of communities ${ }^{f}$ & 9 & 17 & 19 & 17 & 9 \\
\hline Network diameter ${ }^{g}$ & 6 & 9 & 7 & 7 & 6 \\
\hline Average path length ${ }^{h}$ & 1.66 & 2.32 & 1.88 & 2.03 & 1.63 \\
\hline Average degree $^{i}$ & 84.59 & 41.44 & 54.17 & 48.82 & 85.19 \\
\hline Average clustering coefficien $\mathrm{t}^{\mathrm{j}}$ & 0.342 & 0.277 & 0.300 & 0.29 & 0.350 \\
\hline
\end{tabular}

${ }^{a}$ Microbial taxon (at genus level) with at least one significant $(P<0.01$ ) and strong (SparCC $>0.7$ or $<-0.7)$ correlation.

${ }^{\mathrm{b} N u m b e r}$ of connections/correlations obtained by SparCC analysis.

'SparCC-positive correlation ( $>0.7$ with $P<0.01$ ).

dSparCC-negative correlation $(<-0.7$ with $P<0.01)$.

eThe capability of the nodes to form highly connected communities, that is, a structure with high density of between nodes connections (inferred by Gephi).

${ }_{\mathrm{f}}^{\mathrm{A}} \mathrm{A}$ community is defined as a group of nodes densely connected internally (Gephi).

'The longest distance between nodes in the network, measured in number of edges (Gephi).

${ }^{\mathrm{h}}$ Average network distance between all pair of nodes or the average length off all edges in the network (Gephi).

iThe average number of connections per node in the network, that is, the node connectivity (Gephi).

${ }^{j} \mathrm{How}$ nodes are embedded in their neighborhood and the degree to which they tend to cluster together (Gephi).

et al., 2010). Also, the network of the Fox-resistant cultivar presented a more modular structure (Table 1), which is characterized by the presence of different groups of nodes with high numbers of interconnections within, with some degree of independencies between groups (Newman, 2006). A modular structure suggests diversity in species roles and functionality, increasing niche overlap (Poudel et al., 2016). In this sense, a highly connected and modular rhizosphere microbiome could decrease pathogen invasion success if it leads to more efficient consumption for resources (van Elsas et al., 2012; Wei et al., 2015). Also, modularity combined with a short average path length may imply a more prompt response of the microbial community to environmental perturbations (Faust and Raes, 2012).

Based on the network properties, we identified three bacterial groups with more betweenness centrality, which is defined as the number of times a node acts as a bridge along the shortest path between two other nodes (Poudel et al., 2016). In network analysis, the centrality indicates the most important nodes, which may be interpreted as key taxa inside a connected community (Borgatti, 2005). The top three nodes with more centrality were (1) the genus Dyadobacter (phylum Bacteroidetes), (2) a member of the family Comamonadaceae (phylum Proteobacteria) and (3) one taxon affiliated to Acidobacteria Gp16. Interestingly, the genus Dyadobacter was suggested as a potential taxon involved in the suppression of fusarium wilt in the rhizosphere of banana ( $\mathrm{Fu}$ et al., 2017), whereas the family Comamonadaceae was identified as Fox-suppressive bacterial taxon associated with cotton plants (Li et al., 2015). Also, all three key bacterial groups are interconnected in the network of the FoX-resistant cultivar. Key taxa represent nodes that are associated with many others, and a removal of this node may have a large impact on the community structure (Steele et al., 2011). It is important to note that a pathogen invader may manage to displace the key taxa and collapse the network structure (Albrecht et al., 2014).

\section{Conclusions}

The importance of the rhizosphere microbiome in the plant ecosystem functioning has been widely recognized, but traditional approaches of plant breeding do not take the plant microbiome into account. Here, we presented an in-depth analysis of the rhizosphere microbiomes of common bean cultivars with different degrees of Fox resistance. Although Fox resistance is based on plant genetic traits, our data support the hypothesis that breeding for resistance may have unintentionally altered the rhizosphere microbiome composition, altering the frequency of beneficial microorganisms and traits that may contribute to plant growth or assist in protection against the pathogen. Considering that Fox resistance is based on genetic and chemical alterations in the plant, our findings suggest that the observed changes in rhizosphere microbiome may enforce the first line of defense, limiting pathogen invasion by means of a higher abundance of specific microbial groups and functions, high microbial diversity, abundance and a more complex network structure. We also showed that the recruitment of the rhizosphere microbiome is highly dependent on the soil type, where the Amazonian Dark Earth soil provided a richer source of potentially beneficial microorganisms. Future studies will require experimental validation of the putative beneficial effects of 
the identified bacterial taxa. Our results reinforce the importance of understanding the genetic and (bio) chemical mechanisms involved in the interplay between soil type, plant genotype, rhizosphere microbiome, plant growth and plant health. Hence, future plant breeding programs should be directed toward unraveling the molecular basis of interaction between plants and beneficial members of the rhizosphere microbiome. The use of nextgeneration sequencing together with culturedependent approaches are essential to identify and functionally validate the importance of key microbial groups and traits in pathogen suppression. Unraveling the link between specific regions in the plant genome and microbial recruitment is essential for identifying molecular markers in plants that can be used in future breeding programs.

\section{Conflict of Interest}

The authors declare no conflict of interest.

\section{Acknowledgements}

This study was supported by a grant from Fundação de Amparo à Pesquisa do Estado de São Paulo (FAPESP 2014/03217-3, 2015/00251-9). We thank SAM Carbonell and AF Chiorato from Instituto Agronômico de Campinas for the help in selecting the cultivars and providing common bean seeds. Publication 6321 of the Netherlands Institute of Ecology (NIOO-KNAW).

\section{References}

Albrecth M, Padrón B, Bartomeus I, Traveset A. (2014). Consequences of plant invasions on compartmentalization and species' role in plant-pollinator networks. Proc Biol Sci 281: 20140773.

Anderson M. (2001). A new method for non-parametric multivariate analysis of variance. Austral Ecol 26: 32-46.

Anjaiah V, Koedam N, Nowak-Thompson B, Loper JE, Höfte M, Tambong JT et al. (1998). Involvement of phenazines and anthranilate in the antagonism of Pseudomonas aeruginosa PNA1 and Tn5-derivatives towards Fusarium sp. and Pythium sp. Mol PlantMicrobe Interact 11: 847-854.

Aziz RK, Bartels D, Best AA, DeJongh M, Disz T, Edwards RA et al. (2008). The RAST Server: rapid annotation using subsystems technology. BMC Genomics 9: 75 .

Baggett JR, Frazier WA, Vaughn EK. (1965). Tests of Phaseolus species for resistance to Fusarium root rot. Plant Dis Rep 49: 630-633.

Bakker MG, Manter DK, Sheflin AM, Weir TL, Vivanco JM. (2012). Harnessing the rhiosphere microbiome through plant breeding and agricultural management. Plant Soil 360: 1-13.

Bakker MG, Chaparro JM, Manter DK, Vivanco JM. (2015). Impacts of bulk soil microbial community structure on rhizosphere microbiomes of Zea mays. Plant Soil 393: 115-126.

Bastian MHS, Jacomy M. (2009), Gephi: an open source software for exploring and manipulating networks. International AAAI Conference on Weblogs and Social Media: San Jose, CA, USA.

Beckers B, Op De Beeck M, Weyens N, Van Acker R, Van Montagu M, Boerjan W et al. (2016). Lignin engineering infiled-grown poplar trees affects the endosphere bacterial microbiome. Proc Natl Acad Sci USA 113: 2312-2317.

Benjamini Y, Hochberg Y. (1995). Controling the false discovery rate - a practical and powerful approach to multiple testing. J Roy Stat Soc 57: 289-300.

Berg G, Smalla K. (2009). Plant species and soil type cooperatively shape the structure and function of microbial communities in the rhizosphere. FEMS Microbiol Ecol 68: 1-13.

Berg G, Grube M, Schloter M, Smalla K. (2014). Unraveling the plant microbiome: looking back and future perspectives. Front Microbiol 5: 1-7.

Borgatti SP. (2005). Centrality and network flow. Soc Network 27: 55-71.

Brody JR, Kern SE. (2004). Sodium boric acid: Atriz-less, cooler conductive medium for DNA electrophoresis. Biotech 36: 214-216.

Brossi MJL, Mendes LW, Germano MG, Lima AB, Tsai SM. (2014). Assessment of bacterial bph gene in Amazon Dark Earth and their adjacent soils. Plos One 9: e99597.

Bulgarelli D, Rott M, Schlaeppi K, van Themaat EVL, Ahmadinejad N, Assenza F et al. (2012). Revealing structure and assembly cues for Arabidopsis rootinhabiting bacterial microbiota. Nature 488: 91-95.

Bulgarelli D, Garrido-Oter R, Munch PC, Weiman A, Droge J, Pan Y et al. (2015). Structure and function of the bacterial root microbiota in wild and domesticated barley. Cell Host Microb 17: 392-403.

Calba H, Firdaus, Cazevieille P, Thée C, Poss R, Jaillard B. (2004). The dynamics of prótons, aluminium, and calcium in the rhizosphere of maize cultivated in tropical acid soils: experimental study and modelling. Plant Soil 260: 33-46.

Camargo AO, Moniz AC, Jorge JA. Valadares JMAS (2009). Methods of chemical analysis, physical and mineralogical soil. Agronomic Institute of Campinas. Agronomic Institute: Campinas, pp 77.

Caporaso JG, Kuczynski J, Stombaugh J, Bittinger K, Bushman FD, Costello EK et al. (2010). QIIME allows analysis of high-throughput community sequencing data. Nat Methods 7: 335-336.

Carbonell SAM, Chiorato AF, Bolonhezi D, Barros VLNP, Borges WLB, Ticelli M et al. (2014). 'IAC Milênio' Common bean cultivar with high grain quality. Crop Breed Appl Biotechnol 8: 163-166.

Cavaglieri L, Orlando J, Rodríguez MI, Chulze S, Etcheverry M. (2005). Biocontrol of Bacillus subtilis against Fusarium verticillioides in vitro and at the maize root level. Res Microbiol 156: 748-754.

Chapelle E, Mendes R, Bakker PAHM, Raaijmakers JM. (2015). Fungal invasion of the rhizosphere microbiome. ISME J 15: 1751-7362.

Chi-a-Woeng TFC, Bloemberg GV, Mulders IHM, Dekkers LC, Lugtenberg BJJ. (2000). Root colonization by phenazine-1-carboxamide-producing bacterium Pseudomonas chlororaphis PCL1391 is essential for biocontrol of tomato foot and root rot. Mol PlantMicrobe Interact 13: 1340-1345. 
Cole JR, Wang Q, Fish JA, Chai B, McGarrell DM, Sun Y et al. (2014). Ribosomal Database Project: data and tools for high throughput rRNA analysis. Nucleic Acids Res 42: D633-D642.

Dodt M, Roehr JT, Ahmed R, Dieterich C. (2012). FLEXBAR - Flexible barcode and adapter processing for nextgeneration sequencing platforms. Biology 1: 895-905.

Edgar RC. (2010). Search and clustering hundreds of times faster than BLAST. Bioinformatics 26: 2460-2461.

Edgar RC, Haas BJ, Clemente JC, Quince C, Knight R. (2011). UCHIME improves sensitivity and speed of chimera detection. Bioinformatics 27: 2194-2200.

Faith DP. (1992). Conservation evaluation and phylogenetic diversity. Biol Conserv 61: 1-10.

Faust K, Raes J. (2012). Microbial interactions: from networks to models. Nat Rev Microbiol 10: 538-550.

Fisher WD. (1958). On grouping for maximum homogeneity. J Am Stat Assoc 53: 789-798.

Friedman J, Alm EJ. (2012). Inferring correlation networks from genomic survey data. Plos Comput Biol 8: e1002687.

Fu L, Penton CR, Ruan Y, Shen Z, Xue C, Li R et al. (2017). Inducing the rhizosphere microbiome by biofertilizer application to suppress banana Fusarium wilt disease. Soil Biol Biochem 104: 39-48.

Gilard G, Baudino M, Gullino ML, Garibaldi A. (2008). Attempts to control Fusarium root rot of bean by seed dressing. Commun Agric Appl Sci 73: 75-80.

Haba E, Pinazo A, Jauregui O, Espuny MJ, Infante MR, Manresa A. (2003). Physicochemical characterization and antimicrobial properties of rhamnolipids produced by Pseudomonas aeruginosa 47T2 NCBIM 40044. Biotechnol Bioeng 81: 316-322.

Hammer Ø, Harper DAT, Ryan PD. (2001). Past: Paleontological Statistics Software package for education and data analysis. Palaeontol Electron 4: 9.

Heuer H, Krsek M, Baker P, Smalla K, Wellington EMH. (1997). Analysis of actinomycete communities by specific amplification of genes encoding 16S rRNA and gel-electrophoretic separation in denaturing gradients. Appl Environ Microbiol 63: 3233-3241.

Inceoglu O, Salles JF, van Elsas JD. (2012). Soil and cultivar type shape the bacterial community in the potato rhizosphere. Microb Ecol 63: 460-470.

Jones DL, Nguyen C, Finlay RD. (2009). Carbon flow in the rhizosphere: carbon trading at the soil-root interface. Plant Soil 321: 5-33.

Kennedy TA, Naeem S, Howe KM, Knops JMH, Tilman D, Reich P. (2002). Biodiversity as barrier to ecological invasion. Nature 417: 636-638.

Klindworth A, Pruesse E, Schweer T, Peplies J, Quast C, Horn $\mathrm{M}$ et al. (2013). Evaluation of general 16S ribosomal RNA gene PCR primers for classical and next-generation sequencing-based diversity studies. Nucleic Acids Res 41: e1.

Köster J, Rahmann S. (2012). Snakemake - a scalable bioinformatics workflow engine. Bioinformatics 28: 2520-2522.

Kumar P, Dubey RC, Maheshwari DK. (2012). Bacillus strains isolated from rhizosphere showed plant growth promoting and antagonistic activity against phytopatogens. Micobiol Res 167: 493-499.

Latz E, Eisenhauer N, Rall BC, Allan E, Roscher C, Scheu S et al. (2012). Plant diversity improves protection against soil-borne pathogens by foresting antagonistic bacteral communities. J Ecol 100: 597-604.
Lebeis SL, Paredes SH, Lundberg DS, Breakfield N, Gehring J, McDonald M et al. (2015). Salicylic acid modulates colonization of the root microbiome by specific bacterial taxa. Science 349: 860-864.

Leiman PG, Basler M, Ramagopal UA, Bonanno JB, Sauder JM, Pukatzki S et al. (2009). Type VI secretion apparatus and phage tail-associated protein complexes share a common evolutionary origin. Proc Natl Acad Sci USA 106: 4154-4159.

Li X, Zhang Y, Ding C, Jia Z, He Z, Zhang T et al. (2015). Declined soil suppressiveness to Fusarium oxysporum by rhizosphere microflora of cotton in soil sickness. Biol Fertil Soils 51: 935-946.

Lundberg DS, Lebeis SL, Paredes SH, Yourstone S, Gehring J, Malfatti S et al. (2012). Defining the core Arabidopsis thaliana root microbiome. Nature 488: 86-90.

Mace ME, Bell AA, Beckman CH. (1981). Fungal diseases of plants. Academic Press: New York, USA.

Magoč T, Salzberg SL. (2011). FLASH: fast length adjustment of short reads to improve genome assemblies. Bioinformatics 27: 2957-2963.

Mallon CA, van Elsas JD, Salles JF. (2015). Microbial invasions: the process, patterns, and mechanisms. Trends Microbiol 23: 719-729.

Marschner P, Yang CH, Lieberei R, Crowley DE. (2001). Soil and plant specific effects on bacterial community composition in the rhizosphere. Soil Biol Biochem 33: 1437-1445.

Mazurier S, Corberand, Lemanceau P, Raaijmakers JM. (2009). Phenazine antibiotics produced by fluorescent pseudomonads contribute to natural soil suppressiveness to Fusarium wilt. ISME J 3: 977-991.

McDonald D, Clemente JC, Kuczynski J, Rideout JR, Stombaugh J, Wendel D et al. (2012). The Biological Observation Matrix (BIOM) format or: how I learned to stop worrying and love the ome-ome. GigaScience 1: 7.

Mendes LW, Kuramae EE, Navarrete AA, Van Veen JA, Tsai SM. (2014). Taxonomical and functional microbial community selection in soybean rhizosphere. ISME J 8: 1577-1587.

Mendes R, Kruijt M, De Bruijn I, Dekkers E, Van der Voort M, Schneider JHM et al. (2011). Deciphering the rhizosphere microbiome for disease-suppressive bacteria. Science 332: 1097-1100.

Mendes R, Garbeva P, Raaijmakers JM. (2013). The rhizosphere microbiome: significance of plant beneficial, plant pathogenic, and human pathogenic microorganisms. FEMS Microbiol Rev 37: 634-663.

Meyer F, Paarman D, D'Souza M, Olson R, Glass EM, Kubal M et al. (2008). The Metagenomics RAST server - A public resource for the automatic phylogenetic and functional analysis of metagenomes. BMC Bioinformatics 9: 386.

Moore DP, Mason BJ, Maas EV. (1965). Accumulation of calcium in exudate of individual barley roots. Plant Physiol 40: 641-644.

Mukankusi C, Derera J, Melis R, Gibson PT, Butuchara R. (2011). Genetic analysis of resistance to Fusarium root rot in common bean. Euphytica 182: 11-23.

Newcombe RG. (1998). Improved confidence intervals for the difference between binomial proportions based on paired data. Stat Med 17: 2635-2650.

Newman MEJ. (2006). Modularity and community structure in networks. Proc Natl Acad Sci USA 103: 8577-8582. 
Oliveros JC. (2007). VENNY. An interactive tool for comparing lists with Venn Diagrams http://bioinfogp. cnb.csic.es/tools/venny/index.html.

Parks DH, Tyson GW, Hugenholtz P, Beiko RG. (2014). STAMP: Statistical analysis of taxonomic and functional profiles. Bioinformatics 30: 3123-3124.

Pereira AC, Cruz MFA, Paula Júnior TJ, Rodrigues FA, Carneiro JES, Vieira RF et al. (2013). Infection process of Fusarium oxysporum f. sp. phaseoli on resistant, intermediate and susceptible bean cultivars. Tropic Plant Pathol 38: 323-328.

Pérez-Jaramillo JE, Mendes R, Raaijmakers JM. (2015). Impact of plant domestication on rhizosphere microbiome assembly and functions. Plant Mol Biol 90: 635-644.

Philippot L, Raaijmakers JM, Lemanceau P, van der Putten WH. (2013). Going back to the roots: the microbial ecology of the rhizosphere. Nat Rev Microbiol 11: 789-799.

Poudel R, Jumpponen A, Schlatter DC, Paulitz TC, McSpadden Gardener BB, Kinkel LL et al. (2016). Microbiome networks: a systems framework for identifying candidate microbial assemblages for disease management. Phytopathology 106: 1083-1096.

Raaijmakers JM, Mazzola M. (2016). Soil immune responses. Science 352: 1392-1393.

Rengel Z. (2015). Availability of $\mathrm{Mn}, \mathrm{Zn}$ and $\mathrm{Fe}$ in the rhizosphere. J Soil Sci Plant Nutr 15: 397-409.

Ridder-Duine AS, Kowalchuk GA, Gunnewieke PJAK, Smant W, van Veen JA, de Boer W. (2005). Rhizosphere bacterial community composition in natural stands of Carex arenaria (sand sedge) is determined by bulk soil community composition. Soil Biol Biochem 37: 349-357.

Rognes T, Mahé F. xflouris. (2015). vsearch: VSEARCH version 1.0.16 https:/github.com/torognes/vsearch.
Román-Avilés B, Kelly JD. (2005). Identification of quantitative trait loci conditioning resistance to Fusarium root rot in common bean. Crop Sci 45: 1881-1890.

Schneider KA, Kelly JD. (2000). A greenhouse screening protocol for Fusarium root rot in bean. Hortic Sci 35: 1095-1098.

Smith KP, Goodman RM. (1999). Host variation for interactions with beneficial plant-associated microbes. Annu Rev Phytopathol 37: 473-491.

Smith KP, Handelsman J, Goodman RM. (1999). Genetic basis in plants for interactions with diseasesuppressive bacteria. Proc Natl Acad Sci USA 96: 4786-4790.

Steele JA, Countway PD, Xia L, Vigil PD, Beman JM, Kim DY et al. (2011). Marine bacterial, archaeal and protistan association networks reveal ecological linkages. ISME J 5: 1414-1425.

Van Elsas JD, Chiurazzi M, Mallon CA, Elhottova D, Kristufek V, Salles JF. (2012). Microbial diversity determines the invasion of soil by a bacterial pathogen. Proc Natl Acad Sci USA 109: 1159-1164.

Wei Z, Yang T, Friman VP, Xu Y, Shen Q, Jousset A. (2015). Trophic network architecture of root-associated bacterial communities determines pathogen invasion and plant health. Nat Commun 6: 8413 .

Wissuwa M, Mazzola M, Picard C. (2009). Novel approaches in plant breeding for rhizosphererelated traits. Plant Soil 321: 409-430.

Yao H, Wu F. (2010). Soil microbial community structure in cucumber rhizosphere of different resistance cultivars to fusarium wilt. FEMS Microbiol Ecol 72: $456-463$.

Zhou J, Deng Y, Luo F, He Z, Tu Q, Zhi X. (2010). Functional molecular ecological networks. MBio 1: e00169-10.

Supplementary Information accompanies this paper on The ISME Journal website (http://www.nature.com/ismej) 